\title{
Low-density lipoprotein cholesterol and suicidal behaviour in a large sample of first episode psychosis patients
}

Rosa Ayesa-Arriola, Manuel Canal Rivero, Manuel Delgado-Alvarado, Esther Setién-Suero, Jana González-Gómez, Javier Labad, Anthony S. David \& Benedicto Crespo-Facorro

To cite this article: Rosa Ayesa-Arriola, Manuel Canal Rivero, Manuel Delgado-Alvarado, Esther Setién-Suero, Jana González-Gómez, Javier Labad, Anthony S. David \& Benedicto Crespo-Facorro (2017): Low-density lipoprotein cholesterol and suicidal behaviour in a large sample of first episode psychosis patients, The World Journal of Biological Psychiatry, DOI: 10.1080/15622975.2017.1414305

To link to this article: https://doi.org/10.1080/15622975.2017.1414305

View supplementary material $\asymp$

Accepted author version posted online: 13 Dec 2017.

Submit your article to this journal ๒

Q View related articles $\sqsubset$

View Crossmark data $\nearrow$ 
Text $=1547$ words; Tables $=1$; Supplementary table $=1$

\title{
Low-density lipoprotein cholesterol and suicidal behaviour in
}

\section{a large sample of first episode psychosis patients.}

\author{
AUTHORS: \\ Rosa Ayesa-Arriola Ph.D. ${ }^{\text {a, } b, 1^{*}}$ \\ Manuel Canal Rivero M. Sc. ${ }^{a, b, 1}$ \\ Manuel Delgado-Alvarado M. D. Ph.D. ${ }^{\text {b, } c}$ \\ Esther Setién-Suero M. Sc. ${ }^{a, b}$ \\ Jana González-Gómez M.D. Ph.D. ${ }^{a}$ \\ Javier Labad M.D. Ph.D. ${ }^{b, d}$ \\ Anthony S. David M.D., FRCPsych \\ Benedicto Crespo-Facorro M.D. Ph.D. ${ }^{a, b}$
}

\section{Affiliation/Location of Work:}

a Department of Psychiatry, Marqués de Valdecilla University Hospital, IDIVAL. School of Medicine, University of Cantabria, Santander, Spain

b CIBERSAM, Centro Investigación Biomédica en Red Salud Mental, Spain

c Neurology Department, Marqués de Valdecilla University Hospital. Neuroimaging Unit, Valdecilla Biomedical Research Institute, IDIVAL, Santander, Spain

d Department of Psychiatry, Corporació Sanitària Universitaria Parc Taulí, I3PT, UAB, Sabadell, Spain

e Department of Psychosis Studies. Institute of Psychiatry, King's College London. London (UK)

1 These authors contributed equally to this work.

\section{*Corresponding Author:}

Rosa Ayesa Arriola, Ph.D.

Department of Psychiatry, Marqués de Valdecilla University Hospital.

Avda. Valdecilla s/n, 39008

Santander. Spain. Tel: +34-942-202537 Fax: +34-942-203447

E-mail: rayesa@humv.es 


\begin{abstract}
Objectives: Our aims were to confirm the relationship between lipid and lipoprotein concentrations and suicidal behaviour in first episode psychosis (FEP) patients.
\end{abstract}

Methods: Suicidal behaviour was explored in a large FEP sample $(\mathrm{N}=383)$. Baseline lipid profile was compared between those who attempted or completed suicide and those who not presented suicidal behaviour.

Results: Low-density lipoprotein cholesterol (LDL-c) $(\mathrm{OR}=0.99,95 \% \mathrm{CI}=0.98$ 1.00) and depressive symptoms $(\mathrm{OR}=1.15,95 \% \mathrm{CI}=1.06-1.24)$ were significantly related with suicidal behaviour.

Conclusions: Lipid profile test may be considered in the assessment of suicide risk in psychosis and LDL-c an important biological marker.

KEY WORDS: cholesterol; first episode psychosis; lipoproteins; schizophrenia; suicide 


\section{Introduction}

Suicide represents the single most important cause of premature death in first episode psychosis (FEP) patients (Dutta et al. 2010). Earlier stages have been associated with higher risk periods. During the last decades, there has been a growing interest in the role of the low lipid and lipoprotein concentrations in suicidal behaviours, based on the observation of increased number of mortality from suicides, accidents or violencerelated after the use of cholesterol-lowering drugs (De Berardis et al. 2012).

Lipid profile disturbances have been also reported in antipsychotic-naïve patients (Misiak et al. 2017). Moreover, lower total cholesterol (TC) and low-density lipoprotein cholesterol (LDL-c) levels have been found in patients diagnosed by schizophrenia with history of suicidal behaviours (Ainiyet and Rybakowski 2014). In addition, leptin, an adipocyte hormone involved in energy homeostasis, has been shown to be reduced in such patients (Atmaca et al. 2008). Also, significant low TC was found in FEP patients with history of suicidal behaviour (Marcinko et al. 2007). Indeed, Kavoor et al. (2017) found that schizophrenic patients with lower TC, and LDL-c tended to act impulsively and that low lipid profile increased the risk of self-harming behaviours (Kavoor et al. 2017). In a sample of 35 psychiatric patients, low TC and leptin levels were also associated with more violent suicide attempts (Atmaca et al. 2008).

The aims of our study were to confirm the association between lipid profile at baseline and suicidal behaviour occurred during a 3-year follow-up period in a large sample of antipsychotic-naïve FEP patients. Based on previous publications we hypothesized that 1) lower levels of TC, LDL and leptin will be associated with the presence of suicidal behaviours and 2) lower TC and leptin levels would correlate with more lethal methods of suicide attempt. 


\section{Method}

Data from this study came from a large epidemiological and 3-year longitudinal study of FEP program of Cantabria, Spain, (PAFIP) at the University Hospital Marqués de Valdecilla (Pelayo-Terán et al. 2008). The study was approved by the Marqués de Valdecilla University Hospital review board, and written informed consent was obtained from all subjects after complete description of the study. Three hundred and ninety seven patients met the inclusion criteria and were therefore included in the PAFIP program over this period (2001-2010). Baseline sociodemographic and clinical information was recorded for the total sample. Suicidal behaviours, i.e. "potentially selfinjurious behaviour for which the person intended to kill himself/herself" (Silverman, Alan L Berman, et al. 2007; Silverman, Alan L. Berman, et al. 2007), which encompasses suicide attempts and suicide completions, were taken from medical records, which provided information from patients, their families and medical staff. A complete description of the sociodemographic and clinical data have been described in elsewhere (Ayesa-Arriola et al. 2015). The differences between suicide attempters and non-attempters have been shown in supplementary material (see Table S1).

The present study represents a secondary analysis drawn from a subset sample included in Ayesa-Arriola et al. (2015) (Ayesa-Arriola et al. 2015), to specifically test the association between lipid profile and suicide risk. Any participant presented physical illness possible interfering with lipid profile (e.g. hyperthyroidism, malnutrition, chronic anemia, cancer, or severe liver disease) or was under statin therapy. 
Laboratory assessments

TC, high-density lipoproteins cholesterol (HDL-c), LDL-c and leptin were measured. To minimize the effects of diet and lab technique, blood samples were obtained between 8 and 10 am after overnight fasting by the same personnel, in the same setting. Samples were measured by automated methods on an Advia Centaur (SIEMENS, Erlangen, Germany). Serum cholesterol levels were measured by means of immunonephelometry in a Nephelometer Analyzer II (SIEMENS) using the reagents manufactured by SIEMENS. Intra- and inter-assay reproducibility were $<5 \%$ and $<7 \%$, respectively.

Statistical analyses

The Statistical Package for Social Science, version 19.0 (IBM Corp. Released 2010), was used for statistical analyses. All statistical tests were two-tailed, and significance was set at the 0.05 level.

To test if FEP patients with suicidal behaviours presented different lipid profile ANCOVAs analyses were performed adjusted for age, sex and body mass index (BMI). To test if lower lipid profile correlated with high lethal method ANCOVAs analyses were performed adjusted for age, sex and BMI.

Binary logistic regression (backward: conditional) was conducted to identify predictors of suicidal behaviour. Significant variables in the univariate analyses reported in Ayesa-Arriola et al. (Ayesa-Arriola et al. 2015) as well as significant variables found in the present study were included as predictors.

\section{Results}

ANCOVAs analyses confirmed that participants with the presence of suicidal behaviour had significantly lower TC $(\mathrm{F}=4.49 ; \mathrm{p}<0.05)$, LDL $(\mathrm{F}=4.69 ; \mathrm{p}<0.05)$ and 
leptin $(\mathrm{F}=4.04 ; \mathrm{p}<0.05)$ compared to those without suicidal behaviours (Table 1). There was no significant relationship between depressive symptomatology and any lipid component included in this study (information is available upon request).

The regression model contained six independent variables: sex $(\mathrm{p}<0.03)$, depression $(\mathrm{p}<0.01)$, cannabis $(\mathrm{p}<0.04), \mathrm{TC}(\mathrm{p}<0.04)$, LDL-c $(\mathrm{p}<0.03)$ and leptin $(\mathrm{p} \leq 0.05)$ (AyesaArriola et al. 2015). Variance Inflation Factor (VIF) was calculated and there were no VIF's values over 1.01, thus the assumption of multicollineality was not violated. Binary regression analysis showed that high scores in Calgary Depression Scale for Schizophrenia (CDSS) $(\mathrm{OR}=1.15,95 \% \mathrm{CI}=1.06-1.24)$ and low levels of $\mathrm{LDL}$ $(\mathrm{OR}=0.99,95 \% \mathrm{CI}=0.98-1.00)$ were significant independent parameters associated with suicidal behaviours.

\section{Discussion}

Our hypotheses were partially confirmed. In accordance with the first hypothesis, we found that lower baseline LDL-c levels were associated with increased risk of suicide behavior in a 3-year follow-up period after a FEP presentation. Regarding the second hypothesis, we failed to find significant relationship between low lipoprotein concentrations and more violent suicide methods. Although few studies have analysed the relationship between low serum lipid concentrations and violent suicidal behaviours in schizophrenic patients, significant relationship between low levels of TC, LDL-c (Kavoor et al. 2017) and leptin (Atmaca et al. 2008) and more violent suicide methods have been reported. The potential mechanism linking the lipid profile abnormality and elevated suicide risk/impulsivity are still unknown. With the aim of understanding the underlying mechanism, Lalovic et al., (Lalovic et al. 2007) examined cholesterol content in the brain tissue of suicide completers and found that those using violent methods had significantly lower cholesterol content in the frontal cortex compared to non-violent suicide completers, particularly in the orbitofrontal cortex and the ventral 
prefrontal cortex. It has been postulated that deficits in the orbitofrontal cortex or in the ventral prefrontal cortex may result in impaired inhibition of impulses, and in turn, impulsive aggression and violent or suicidal behaviour. Lower cholesterol in the frontal cortex of violent suicide completers reported in this study supported the idea that deficits in specific areas within the frontal cortex may be linked to impaired inhibition of violent behaviours and suicide. We found a trend towards significant differences between those who used violent methods and those who used non-violent methods in the levels of TC $(157.06 \pm 25.24$ vs. $170.85 \pm 27.46, \mathrm{p}=0.07)$ and leptin $(4.87 \pm 5.70$ vs. $6.39 \pm 5.34, \mathrm{p}=0.07)$. Finally, not surprisingly, depression was associated with suicidal behaviour as extendedly previously reported (Challis et al. 2013).

A diathesis-stress model has been proposed to explain the relationship between lipoprotein concentrations and suicidal behaviour (Perez-Rodriguez et al. 2008). Diathesis model suggested the presence of a low lipid profile as a trait factor related to aggression/impulsivity. Serum lipids levels might influence behaviours such as impulsivity, aggression and suicide through their influences on serotonergic transmission in the brain. Serum lipids could play a role in the production of the myelin sheath, in transmembrane exchange, enzyme function, synthesis of steroid hormones and neurotransmitter receptor expression (De Berardis et al. 2012). A possible explanations is that leptin regulates dopamine responses to salient stress stimuli not directly linked to food. On the other hand, stress model sets forth that a low lipid profile could set off the suicidal behaviour. In our study, we found that exclusively low LDL-c level was independently associated with suicidal behaviour, which, according to the previous model mentioned, could act as a trait factor associated with aggression/impulsivity or as a triggering factor of the suicidal behaviour. 
Some limitations need to be considered. Firstly, the design of the study did not allow to capture the dynamics of the lipids and their influence on suicidal behaviour. Secondly, although serotoninergic transmission, depends in part on brain cholesterol, experimental animal studies have demonstrated that brain cholesterol is relatively insulated from changes in circulating cholesterol. Thus, should be interpreted with caution. However, to the best of our knowledge no previous study has assessed the association between lipid profile with suicidal behaviours and suicidal methods in a such large sample of antipsychotic-naïve FEP patients.

In conclusion, the role of low LDL-c in suicidal behaviour is supported by the results of this study. Suicide accounts for a large percentage of deaths in patients with FEP and other psychotic disorders and is an important health concern. Suicidal behaviour is far from clear and seems that it depends on different cultural, social, individual and biological factors in a complex interrelated relationship. However, most of evidence suggests that risk of suicidal behaviour is relatively high in FEP patients, being early intervention and correct management in FEP patients critical to minimize their suicidality (Pompili et al. 2011). The identification of a low lipid profile at baseline may contribute to improve accuracy in detecting high-risk patients and to develop or strengthen suicide prevention strategies in FEP patients during the early stages of the disorder.

\section{Acknowledgements}

The authors wish to thank all PAFIP research team and all patients and family members who participated in the study. 


\section{Funding Sources}

This work was supported by the Instituto de Salud Carlos III (PI14/00639 and PI14/00918) and Fundación Instituto de Investigación Marqués de Valdecilla (NCT0235832 and NCT02534363). No pharmaceutical company has financially supported the study.

\section{Disclosure Statement}

None to declare.

\section{References}

Ainiyet B, Rybakowski JK. 2014. Suicidal behavior in schizophrenia may be related to low lipid levels. Med. Sci. Monit. [Internet] 20:1486-90. Available from:

http://www.pubmedcentral.nih.gov/articlerender.fcgi?artid=4148359\&tool=pmcentrez\& rendertype $=$ abstract

Atmaca M, Kuloglu M, Tezcan E, Ustundag B. 2008. Serum leptin and cholesterol values in violent and non-violent suicide attempters. Psychiatry Res. 158:87-91.

Ayesa-Arriola R, Alcaraz EG, Hernández BV, Pérez-Iglesias R, López Moríñigo JD, Duta R, et al. 2015. Suicidal behaviour in first-episode non-affective psychosis: Specific risk periods and stage-related factors. Eur. Neuropsychopharmacol. 25:2278-2288.

De Berardis D, Marini S, Piersanti M, Cavuto M, Perna G, Valchera A, et al. 2012. The Relationships between Cholesterol and Suicide: An Update. ISRN Psychiatry [Internet] 2012:1-6. Available from: https://www.hindawi.com/journals/isrn/2012/387901/

Challis S, Nielssen O, Harris A, Large M. 2013. Systematic meta-analysis of the risk factors for deliberate self-harm before and after treatment for first-episode psychosis. Acta Psychiatr. Scand. 127:442-454. 
Dutta R, Murray RM, Hotopf M, Allardyce J, Jones PB, Boydell J. 2010. Reassessing the long-term risk of suicide after a first episode of psychosis. Arch. Gen. Psychiatry 67:1230-1237.

IBM Corp. Released. 2010. IBM SPSS Statistics for Windows, Version 19.0.

Kavoor AR, Mitra S, Kumar S, Sisodia AK, Jain R. 2017. Lipids, aggression, suicidality and impulsivity in drug-naïve/drug-free patients of schizophrenia. Asian J. Psychiatr. [Internet] 27:129-136. Available from:

http://www.sciencedirect.com/science/article/pii/S1876201816306049

Lalovic A, Levy E, Luheshi G, Canetti L, Grenier E, Sequeira A, et al. 2007.

Cholesterol content in brains of suicide completers. Int. J. Neuropsychopharmacol. 10:159-166.

Marcinko D, Pivac N, Martinac M, Jakovljević M, Mihaljević-Peles A, Muck-Seler D. 2007. Platelet serotonin and serum cholesterol concentrations in suicidal and nonsuicidal male patients with a first episode of psychosis. Psychiatry Res. [Internet] 150:105-8. Available from:

http://www.sciencedirect.com/science/article/pii/S0165178106002617

Misiak B, Stańczykiewicz B, Łaczmański Ł, Frydecka D. 2017. Lipid profile disturbances in antipsychotic-naive patients with first-episode non-affective psychosis: A systematic review and meta-analysis. Schizophr. Res. [Internet]. Available from: http://www.sciencedirect.com/science/article/pii/S092099641730169X

Pelayo-Terán JM, Pérez-Iglesias R, Ramírez-Bonilla ML, González-Blanch C, Martínez-García O, Pardo-García G, et al. 2008. Epidemiological factors associated with treated incidence of first-episode non-affective psychosis in Cantabria: Insights from the Clinical Programme on Early Phases of Psychosis. Early Interv. Psychiatry 
2:178-187.

Perez-Rodriguez MM, Baca-Garcia E, Diaz-Sastre C, Garcia-Resa E, Ceverino A, SaizRuiz J, et al. 2008. Low serum cholesterol may be associated with suicide attempt history. J. Clin. Psychiatry 69:1920-1927.

Pompili M, Serafini G, Innamorati M, Lester D, Shrivastava A, Girardi P, et al. 2011. Suicide risk in first episode psychosis: a selective review of the current literature. Schizophr. Res. [Internet] 129:1-11. Available from: http://www.ncbi.nlm.nih.gov/pubmed/21530179 Silverman MM, Berman AL, Sanddal ND, O’Carroll PW, Joiner TE. 2007. Rebuilding the tower of Babel: a revised nomenclature for the study of suicide and suicidal behaviors. Part 1: Background, rationale, and methodology. Suicide Life. Threat. Behav. [Internet] 37:248-63. Available from: http://www.ncbi.nlm.nih.gov/pubmed/17579539\%5Cnhttp://www.ncbi.nlm.nih.gov/pub $\operatorname{med} / 17579538$

Silverman MM, Berman AL, Sanddal ND, O’Carroll PW, Joiner TE. 2007. Part 2:

Suicide-related ideations, communications, and behaviors. Suicide Life. Threat. Behav. $37: 264-277$. 
Table1.

Differences between FEP patients with suicidal and non-suicidal behaviors

\begin{tabular}{|c|c|c|c|c|c|}
\hline & $\begin{array}{l}\text { Total sample } \\
(n=383)\end{array}$ & $\begin{array}{l}\text { FEP patients } \\
\text { with suicidal } \\
\text { behaviors } \\
(n=57)\end{array}$ & $\begin{array}{l}\text { FEP patients } \\
\text { without suicidal } \\
\text { behaviors }(n=326)\end{array}$ & $\begin{array}{l}\text { Test } \\
\text { statistic }^{a}\end{array}$ & $p$ \\
\hline $\begin{array}{l}\text { Total Cholesterol } \\
(\mathrm{mg} / \mathrm{dL})^{\mathrm{b}}\end{array}$ & $176.37 \pm 39.14$ & $162 \pm 60.46$ & $176,38 \pm 40.59$ & $\mathrm{~F}=4.49$ & $0.04 *$ \\
\hline HDL-c $(\mathrm{mg} / \mathrm{dL})^{\mathrm{b}}$ & $51.65 \pm 14.75$ & $46.98 \pm 13.89$ & $52.38 \pm 14.77$ & $\mathrm{~F}=3.12$ & 0.08 \\
\hline LDL-c (mg/dL) $)^{b}$ & $106.89 \pm 32.75$ & $96.09 \pm 23.61$ & $108.58 \pm 33.68$ & $\mathrm{~F}=4.69$ & $0.03^{*}$ \\
\hline Leptin (mg/dl) $)^{b}$ & $7.45 \pm 7.68$ & $5.52 \pm 5.44$ & $7.74 \pm 7.93$ & $\mathrm{~F}=4.04$ & $0.05^{*}$ \\
\hline Triglycerides $(\mathrm{mg} / \mathrm{dL})^{b}$ & $84.52 \pm 42.41$ & $91.49 \pm 40.21$ & $83.39 \pm 42.72$ & & .27 \\
\hline Glucose $(\mathrm{mg} / \mathrm{dL})^{b}$ & $87.24 \pm 17.50$ & $90.31 \pm 38.09$ & $86.73 \pm 10.80$ & & .14 \\
\hline BMI & $23.18 \pm 3.56$ & $23.15 \pm 3.02$ & $23.18 \pm 3.65$ & $t=0.06$ & 0.96 \\
\hline
\end{tabular}

FEP: First Episode of Psychosis; DUP: Duration Untreated Psychosis; BPRS: Brief Psychiatric Rating Scale; SAPS: Scale for the Assessment of Positive Symptoms; SANS: Scale for the Assessment of Negative Symptoms; CDSS: Calgary Depression Scale for Schizophrenia; SUMD: Scale of Unawareness of Mental Disorder; HDL-c: High-Density Lipoproteins- cholesterol; LDL-c: LowDensity Lipoproteins-cholesterol; BMI: Body Mass Index.

${ }^{\text {a }}$ Differences between patients with suicide attempts and without suicide attempts analyzed with chi-square, T-test and ANCOVA.

${ }^{\mathrm{b}}$ Controlled for sex, age and BMI.

$p<0.05$

Binary regression model in subsequent blocks (backward: conditional) with suicidal behavior (no/yes) as the dependent variable $(n=332)$

\begin{tabular}{lllllll}
\hline & $\boldsymbol{B}$ & $\boldsymbol{S E}$ & Wald & $\boldsymbol{p}$ & OR & 95\% CI \\
\hline CDSS & 0.14 & 0.04 & 11.39 & 0.01 & 1.15 & $1.06-1.24$ \\
\hline LDL-c & -0.01 & 0.01 & 4.40 & 0.04 & 0.99 & $0.98-1.00$
\end{tabular}

$\mathrm{x}^{2}(3)=18.58, \mathrm{p} \leq 0.01, \mathrm{R}^{2}$ Cox and Snell $=0.05, \mathrm{R}^{2}$ Nagelkerke $=0.1$

CDSS: Calgary Depression Scale for Schizophrenia; LDL-c: Low-Density Lipoproteins-cholesterol

$p<0.05$ 